\title{
AMOSTRAGEM PARA DIAGNOSE DO ESTADO NUTRICIONAL DE MANGUEIRAS ${ }^{1}$
}

\author{
DANILO EDUARDO ROZANE ${ }^{2,6}$, WILLIAM NATALE $^{3,7}$, RENATO DE MELLO PRADO $^{4,7}$, JOSÉ CARLOS BARBOSA $^{5}$
}

RESUMO - Dentre todas as etapas que permeiam um laudo foliar, ainda a amostragem continua sendo a mais sujeita a erros. O presente trabalho teve como objetivo determinar o tamanho de amostras foliares e a variação do erro amostral para coleta de folhas de pomares de mangueiras. O experimento contou com delineamento inteiramente casualizado, com seis repetições e quatro tratamentos, que constaram da coleta de uma folha, em cada uma das quatro posições cardeais, em 5; 10; 20 e 40 plantas. Com base nos resultados dos teores de nutrientes, foram calculados as médias, variâncias, erros-padrão das médias, o intervalo de confiança para a média e a porcentagem de erro em relação à média, através da semi-amplitude do intervalo de confiança expresso em porcentagem da média. Concluiu-se que, para as determinações químicas dos macronutrientes, 10 plantas de mangueira seriam suficientes, coletando-se uma folha nos quatro pontos cardeais da planta. Já para os micronutrientes, seriam necessárias, no mínimo, 20 plantas e, se considerarmos o Fe, seria necessário amostrar, pelo menos, 30 plantas.

Termos de indexação: diagnose foliar, Mangifera indica, nutrição mineral

\section{SIZE OF SAMPLES FOR NUTRITIONAL STATUS ASSESSMENT OF MANGO TREES}

\begin{abstract}
Of all the processes used in foliar evaluation, sampling is currently still the most subject to error. To corroborate this, the present study aimed to determine the size of foliar samples and error variation of samples collected from mango tree leaves. The study used a completely randomized design with six repetitions and four different treatments which consisted of a leaf from each of the cardinal points from 5, 10, 20 and 40 different trees. Mean variation, mean standard error, mean confidence interval and error percentage compared to mean values were calculated based on level of nutrients. Results were calculated by using the semi-amplitude of the interval of confidence expressed as mean percentages. Obtained results led to the conclusion that the chemical evaluation of macro-nutrients from 10 mango plants would be sufficient for assessment. However, for assessment of micro-nutrients, the sampling of 20 plants would be necessary, and, when considering Fe, a total of 30 plants should be used.
\end{abstract}

Index terms: leaf diagnosis, Mangifera indica, mineral nutrition.

\section{INTRODUÇÃO}

A mangueira (Mangifera indica L.) é uma frutífera pertencente à família Anacardiaceae, originária da Ásia, do centro Indiano e do subcentro Indo-Malaio (Pinto, 2004). Apesar de a manga ser uma das principais frutas tropicais cultivadas no Brasil, a produtividade média dos pomares (cerca de $8 \mathrm{t} \mathrm{ha}^{-1}$ ) é baixa, encontrando-se próxima ao rendimento médio mundial, não sendo naturalmente um bom volume de produção (Lirio, 2004). Entretanto, continua sendo a fruta tropical que mais contribui com as exportações brasileiras de frutas frescas (Prado, 2004), apesar de ainda pequena, se comparado ao total produzido (Pinto, 2004), necessitando, desta maneira, de atenção e aprimoramento em suas tecnologias de manejo, principalmente quanto aos aspectos ligados à nutrição, calagem e adubação.

Em mangueiras, a análise química de folhas auxilia no conhecimento do estado nutricional da cultura, avalia e calibra o resultado das adubações realizadas com base na interpretação da análise de solo e, ainda, é empregada para estabelecer a recomendação da adubação nitrogenada. Marschner (1995) ressalta que, para frutíferas, a análise foliar se torna ainda mais útil que a análise de solo.

Um dos principais condicionantes ao êxito da correta interpretação do estado nutricional das plantas está na amostragem, em especial quanto ao número de plantas a serem amostradas por talhão, para se obter confiabilidade na diagnose.

A indicação mais aceita para a época de amostragem de folhas da mangueira é no pleno florescimento do pomar (Thakur et al., 1981; Avilan \& Carmelo, 1990). Na literatura nacional, apesar de poucos estudos e de divergências entre os resultados, há indicações do número de folhas, consideradas adequadas para compor a amostra. Guimarães (1982) recomenda $60-80$ folhas por talhão homogêneo, provenientes de 10 plantas, após o período de colheita. Malavolta (1992), Quaggio et al. (1997) e Siqueira et al. (1999) indicam a coleta de 80, 80 e 60 folhas, respectivamente, provenientes de 20 plantas, durante o pleno florescimento da

(Trabalho 176-06). Recebido em : 08-11-2006. Aceito para publicação em : 13-04-2007.

2 Engenheiro Agrônomo, Doutorando, Depto. Solos e Adubos, Universidade Estadual Paulista, Fac. de Ciências Agrárias e Veterinárias. Via de Acesso Prof. Paulo Donato Castellane, s/n. 14870-000, Jaboticabal-SP. E-mail: danilorozane@yahoo.com.br

${ }^{3}$ Professor Adjunto, Depto. de Solos e Adubos, Universidade Estadual Paulista, Fac. de Ciências Agrárias e Veterinárias. E-mail: natale@fcav.unesp.br

${ }^{4}$ Professor Doutor, Depto. de Solos e Adubos, Universidade Estadual Paulista, Fac. de Ciências Agrárias e Veterinárias. E-mail: rmprado@fcav.unesp.br

${ }_{5}$ Professor Titular, Depto. Ciências Exatas, Universidade Estadual Paulista, Fac. de Ciências Agrárias e Veterinárias. E-mail: jcbarbosa@fcav.unesp.br

${ }^{6}$ Bolsista da FAPESP.

7 Bolsista do CNPq. 
cultura. Para plantações na Costa Rica, Ponchner et al. (1993) indicam quantidades menores, 30 folhas por talhão homogêneo.

Tendo em vista os poucos estudos sobre amostragem de folhas em mangueiras, buscaram-se subsídios em experimentação com outras culturas, sendo relatadas a seguir. Assim, Natale et al. (1994) e Dahiya \& Joon (1995) verificaram que, para goiabeira, a influência do número de folhas coletadas afetava a composição da amostra em termos nutricionais, concluindo que deve ser de 30 pares de folhas por talhão homogêneo. Sodré et al. (2001) indicam para o cacaueiro que a coleta de folhas em 10 plantas teria representatividade suficiente para a análise foliar.

Para o eucalipto, Lamb (1976) concluiu que, para uma estimativa da média, com um intervalo de confiança de 5 a 10\% de probabilidade, seria necessária a coleta de folhas de quatro árvores para nutrientes com menor variabilidade, como o nitrogênio e o fósforo; para o magnésio, seriam necessárias 11 plantas e, para o cálcio e o enxofre, mais de 20 plantas. No entanto, apesar da grande variação entre os micronutrientes, 37 árvores seriam suficientes para manter o intervalo de confiança acima citado. Mead (1984) considera como recomendação geral, para árvores perenes, a obtenção de uma amostra composta a partir de 20 plantas.

Para a cana-de-açúcar, os trabalhos realizados por Orlando Filho \& Campos (1975 a, b), em cana-planta e cana-soca, respectivamente, revelam que o número de folhas +3 , necessário para representar um hectare, é de apenas duas folhas; entretanto, indicam um mínimo de 15 folhas para compor a matéria seca necessária para a realização das análises laboratoriais.

Pode-se afirmar que a amostragem de tecido vegetal mais adequada é aquela que represente o melhor possível a área a ser avaliada, com um mínimo de plantas amostradas para atender a esse objetivo. Assim, a análise estatística auxilia na indicação de um número de plantas suficiente para reduzir a variação dos resultados a um nível aceitável, ou seja, reduzindo o número de graus de liberdade que exprimem o acaso. Com isso, tem-se uma amostra representativa da população para que os resultados da análise foliar tenham validação técnica e científica.

Buscando maiores esclarecimentos sobre o assunto, objetivou-se determinar, para a mangueira, o número de plantas a serem amostradas para diagnose do estado nutricional da mangueira e avaliar a variação do erro amostral para coleta de folhas utilizadas em diagnóstico nutricional dos pomares.

\section{MATERIAL E MÉTODOS}

O estudo foi realizado em um pomar comercial de mangueiras, com oito anos, variedade Palmer, não-irrigado, localizado no município de Vista Alegre do Alto-SP, com coordenadas geográficas $21^{\circ} 15^{\prime} 22^{\prime}$ " latitude sul, 48¹8'58', longitude oeste e altitude de $603 \mathrm{~m}$. Segundo a classificação de Köppen, o clima local é do tipo Cwa subtropical com inverno curto, moderado e seco, verão quente e chuvoso, caracterizando duas estações climáticas distintas.

O solo do pomar é um Latossolo Vermelho-Amarelo distrófico, segundo a classificação da Embrapa (1999). Realizouse na área de estudo a coleta de 20 subamostras de terra, na projeção da copa das mangueiras, a fim de compor a amostra composta na camada de $0-20 \mathrm{~cm}$, que foi analisada quimicamente (Raij et al., 2001) para determinar a fertilidade do solo (Tabela 1).

De acordo com as condições de solo adequadas, propostas por Quaggio et al. (1997b), o presente experimento (Tabela 1) apresenta-se com fertilidade média para saturação de bases e para fósforo e alta para potássio. De acordo com Raij et al. (1997), os valores foram baixos para enxofre, boro e zinco e altos para cobre, ferro e manganês.

O delineamento experimental empregado foi o inteiramente casualizado, com seis repetições e quatro tratamentos, que constaram da coleta de uma folha, em cada uma das quatro posições cardeais em 5; 10; 20 e 40 plantas, ou seja, 20; 40; 80 e 160 folhas nos tratamentos $1 ; 2 ; 3$ e 4, respectivamente. Seguiuse a recomendação proposta por Quaggio et al. (1997a), coletandose as folhas no meio do último fluxo de vegetação, no terço médio da planta, no início do pleno florescimento, o qual ocorreu no final do mês de julho para a referida região.

O material foi lavado e seco em estufa de ventilação forçada de ar a $65^{\circ} \mathrm{C}$, até atingir massa constante. As amostras secas foram moídas em moinho tipo Wiley, para posterior análise química de macro e micronutrientes, seguindo a metodologia descrita por Bataglia et al. (1983).

Com base nos resultados dos teores de nutrientes, foram calculadas as médias, variâncias, erros-padrão das médias, o intervalo de confiança para a média e a porcentagem de erro em relação à média, através da semi-amplitude do intervalo de confiança (a um coeficiente de 95\% de confiança) expresso em porcentagem da média, dado por:

$$
\% \text { de Erro }=\frac{t s(\hat{m})}{\hat{m}} \times 100
$$

onde,

$t$ é o valor da distribuição $t$ de Student, a $5 \%$ de probabilidade;

$s(\hat{m})$ é o erro-padrão da média, e

$\hat{m}$ é a estimativa da média amostral.

\section{RESULTADOS E DISCUSSÃO}

A Tabela 2 reúne as indicações da literatura sobre os teores de nutrientes considerados adequados para a mangueira. A Tabela 3 apresenta a estatística descritiva e a porcentagem de erro na estimativa da média amostral, além dos resultados médios das análises químicas das folhas de mangueiras com seus limites de variação, nos diferentes números de plantas por amostra, obtidos no presente estudo.

Verifica-se que os teores de fósforo e enxofre estão dentro da faixa indicada como ideal na literatura; porém o nitrogênio e o potássio estão acima (Tabelas 2 e 3). Salienta-se que estas variações no teor foliar de $\mathrm{N}$ e K podem ocorrer de um ano para outro e também a idade da planta influencia no teor de nutrientes, conforme indica Klein (1980) em pomares de maçã. Medeiros et al. (2004) relatam, ainda, que, no florescimento, os teores foliares 
de N e K são comumente superiores aos das outras fases fenológicas da cultura da manga. Os teores de $\mathrm{Ca}$ e $\mathrm{Mg}$ apresentam-se abaixo dos indicados por Quaggio et al. (1997) e Malavolta et al. (1997), mas concordam com a faixa proposta por Medeiros et al. (2004) para a mangueira. Comparando-se com os valores relatados por Young \& Koo (1969), citado por Guimarães (1982), o teor de cálcio está acima do adequado, e o magnésio, dentro da faixa.

Em relação aos micronutrientes, os baixos teores de $\mathrm{B}$ e $\mathrm{Zn}$ e o alto teor de $\mathrm{Mn}$ nas folhas podem estar relacionados à concentração destes nutrientes no solo, uma vez que as concentrações dos elementos determinados no solo (Tabela 1) são interpretados como baixo para B e $\mathrm{Zn}$ e alto para $\mathrm{Mn}$ (Quaggio et al., 1997). Já os teores de Cu concordam com a faixa indicada por Quaggio et al. (1997) (Tabela 2). A concentração de Fe, apesar de adequada no solo (Quaggio et al., 1997), encontra-se abaixo da faixa de teores foliares indicada como ideal (Tabela 2). Os teores de micronutrientes em folhas de mangueira sofrem grandes oscilações entre pomares (Medeiros et al., 2004). Além disso, as diferenças nos teores do tecido vegetal, entre o presente trabalho e a literatura, devem-se, provavelmente, às condições de cultivo, manejo do solo, as épocas e os estágios fenológicos e as diferentes variedades de mangueira.

A análise estatística realizada com os dados coletados

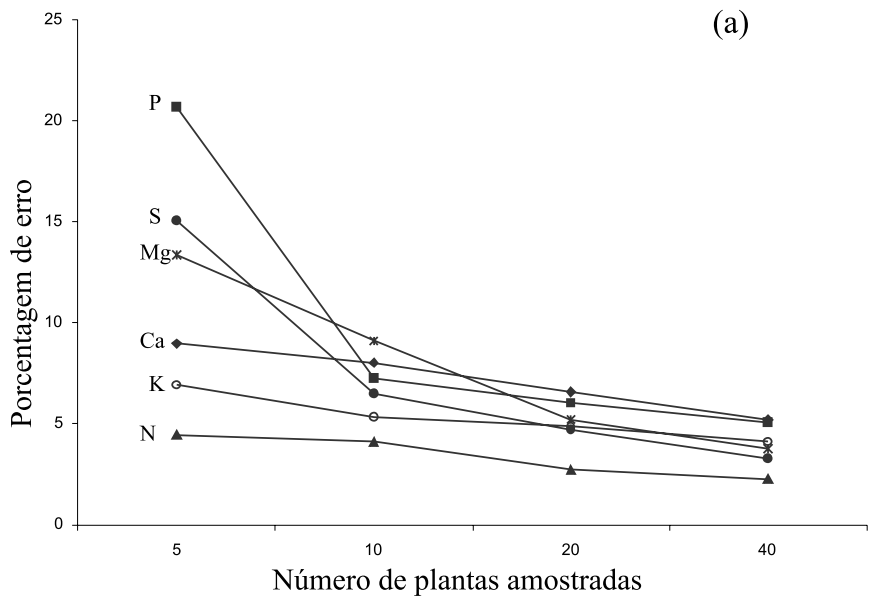

no presente estudo está descrita na Tabela 3. Os erros obtidos em função do número de plantas amostradas foram apresentados nas Figuras 1a, b para macro e micronutrientes, respectivamente.

Os maiores erros amostrais foram detectados quando se coletaram amostras em menor número de plantas (5), diminuindo o erro com o aumento do número de mangueiras amostradas. $\mathrm{O}$ mesmo comportamento ocorreu para o coeficiente de variação (Tabela 3).

Para florestas de eucalipto, Lamb (1976) propõe que um erro entre 5 e $10 \%$ seria aceitável, indicando ser necessário amostrar 10 plantas quando se quer analisar apenas os macronutrientes, mas 20 árvores quando se deseja analisar, também, os micronutrientes. Para a macieira, Holland et al. (1967) consideram aceitável um erro de $20 \%$, quando se coletam 100 folhas proveniente de 5 árvores, erro que provocaria uma amplitude de variação de $\pm 0,22 ; 0,01 ; 0,17 ; 0,18$ e $0,03 \%$ nos teores foliares de $\mathrm{N}, \mathrm{P}, \mathrm{K}, \mathrm{Ca}$ e $\mathrm{Mg}$, respectivamente.

Mead (1984) considera para Pinus radiata um erro aceitável entre 5 e $10 \%$ para a maioria dos macronutrientes e o $\mathrm{Cu}$; mas para o $\mathrm{Ca}$ e os demais micronutrientes, é norma que se tenham erros maiores, decorrentes do maior coeficiente de variação entre árvores. Knight (1978) considera que grande parte da discrepância nos teores foliares em Pinus está associada à diferença clonal, embora essa grandeza variasse menos de $2 \%$ para o $\mathrm{Ca}$, mas $48 \%$ para o $\mathrm{B}$.

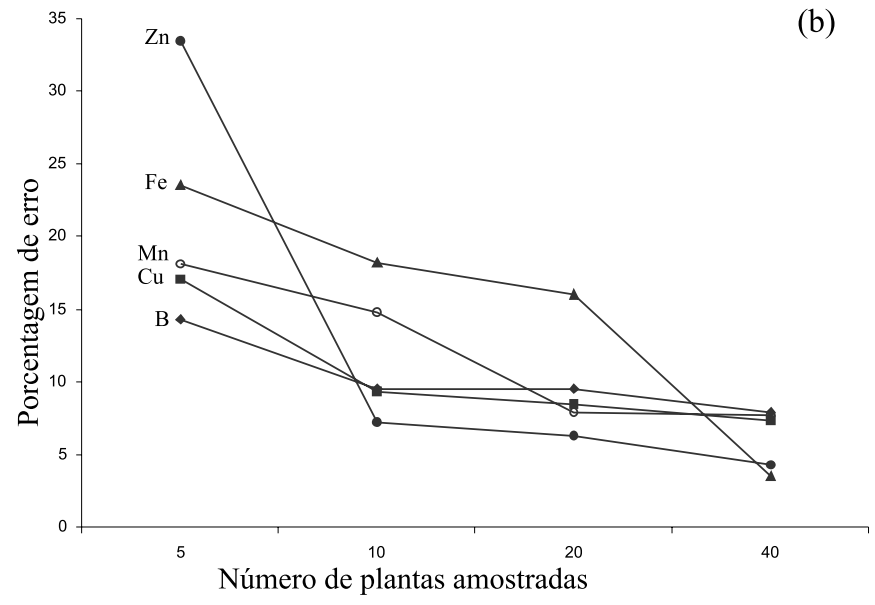

FIGURA 1 - Porcentagem de erro amostral de macro e micronutrientes, em função do número de plantas a serem coletadas por talhão homogêneo, em pomar de mangueira variedade Palmer, em regime não-irrigado.

TABELA 1- Atributos químicos do solo do talhão de mangueiras em estudo.

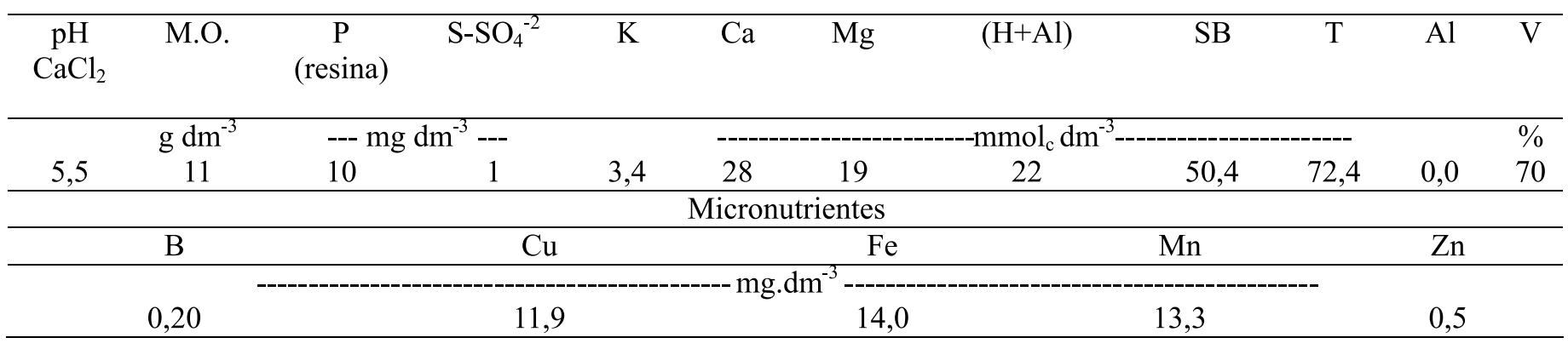


TABELA 2 - Teores foliares de nutrientes considerados adequados para a mangueira, em função da folha-diagnóstica.

\begin{tabular}{|c|c|c|c|c|c|c|c|c|c|c|}
\hline $\mathrm{N}$ & $\mathrm{P}$ & $\mathrm{K}$ & $\mathrm{Ca}$ & $\mathrm{Mg}$ & $\mathrm{S}$ & B & $\mathrm{Cu}$ & $\mathrm{Fe}$ & $\mathrm{Mn}$ & $\mathrm{Zn}$ \\
\hline \multicolumn{6}{|c|}{ 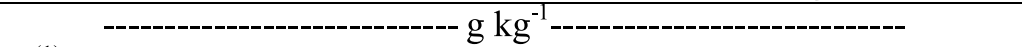 } & \multicolumn{5}{|c|}{-------------------- mg kg'-1------------------- } \\
\hline${ }^{(1)} 10-15$ & $0,8-1,8$ & $3-8$ & $2-5$ & $1,5-4$ & - & - & - & - & - & - \\
\hline (2) $12-14$ & $0,8-1,6$ & $5-10$ & $20-35$ & $2,5-5$ & $0,8-1,8$ & $50-100$ & $10-50$ & $50-200$ & $50-100$ & $20-40$ \\
\hline (3) $10-12$ & $0,8-1,2$ & $4-5$ & $28-34$ & $5-8$ & $1,5-1,8$ & 30 & 30 & 70 & 120 & 90 \\
\hline${ }^{(4)} 12-13$ & $1,2-1,4$ & $4-6$ & $30-33$ & $5-6$ & $1,6-1,8$ & 30 & 30 & 70 & 120 & 90 \\
\hline (5) $10,4-10,9$ & $0,7-0,9$ & $5,3-10,2$ & $9,8-31,6$ & $1,6-2,9$ & - & - & $24-224$ & $190-231$ & $31-370$ & $40-60$ \\
\hline
\end{tabular}

(1) Young \& Koo (1969), citado por Guimarães (1982). Folhas de lançamentos com 4-6 meses, amostradas entre a $3^{\mathrm{a}}$ e $6^{\mathrm{a}}$ folhas.

(2) Quaggio et al. (1997). Folhas do meio do último fluxo de vegetação de ramos com flores.

(3) Malavolta et al. (1997). $2^{\mathrm{a}}$ ou $3^{\mathrm{a}}$ folha na base da panícula, em ramos com frutos.

(4) Malavolta et al. (1997). $2^{a}$ ou $3^{a}$ folha na base da panícula, em ramos sem frutos.

${ }^{(5)}$ Medeiros et al. (2004). Entre a $3^{\text {a }}$ e a $6^{a}$ folhas do último fluxo vegetativo de ramos com flores.

TABELA 3 - Estatísticas descritivas e porcentagem de erro na estimativa da média amostral para macro e micronutrientes em folhas de mangueira, variedade Palmer, sob regime não- irrigado, em função do número de folhas amostradas.

\begin{tabular}{|c|c|c|c|c|c|c|c|c|c|c|c|}
\hline Número de plantas amostradas & $\mathrm{N}$ & $\mathrm{P}$ & $\mathrm{K}$ & $\mathrm{Ca}$ & $\mathrm{Mg}$ & $\mathrm{S}$ & B & $\mathrm{Cu}$ & $\mathrm{Fe}$ & $\mathrm{Mn}$ & $\mathrm{Zn}$ \\
\hline \multicolumn{12}{|l|}{5 Plantas } \\
\hline$m$ & 16,03 & 1,08 & 10,93 & 11,00 & 1,82 & 1,37 & 13,67 & 9,83 & 36,17 & 833,33 & 16,17 \\
\hline $\mathrm{s}^{2}$ & 0,47 & 0,05 & 0,51 & 0,89 & 0,05 & 0,04 & 3,47 & 2,57 & 65,77 & 20666,67 & 26,57 \\
\hline s & 0,68 & 0,21 & 0,72 & 0,94 & 0,23 & 0,20 & 1,86 & 1,60 & 8,11 & 143,76 & 5,15 \\
\hline $\mathrm{s}(\hat{m})$ & 0,28 & 0,09 & 0,29 & 0,38 & 0,09 & 0,08 & 0,49 & 0,65 & 3,31 & 58,69 & 2,10 \\
\hline LI & 15,32 & 0,86 & 10,18 & 10,01 & 1,57 & 1,16 & 11,71 & 8,15 & 27,66 & 682,47 & 10,76 \\
\hline LS & 16,75 & 1,31 & 11,69 & 11,99 & 2,06 & 1,57 & 15,62 & 11,51 & 44,68 & 984,20 & 21,58 \\
\hline E & 4,47 & 20,70 & 6,89 & 8,99 & 13,38 & 15,10 & 14,30 & 17,10 & 23,53 & 18,10 & 33,46 \\
\hline $\mathrm{CV}$ & 4,26 & 19,73 & 6,56 & 8,57 & 12,75 & 14,39 & 13,62 & 16,29 & 22,42 & 17,25 & 31,88 \\
\hline \multicolumn{12}{|l|}{10 Plantas } \\
\hline$m$ & 16,20 & 1,08 & 11,22 & 11,73 & 1,85 & 1,35 & 13,33 & 10,00 & 35,50 & 975,00 & 13,00 \\
\hline $\mathrm{s}^{2}$ & 0,40 & 0,01 & 0,33 & 0,81 & 0,04 & 0,01 & 1,47 & 0,80 & 37,90 & 18750,00 & 0,80 \\
\hline s & 0,63 & 0,08 & 0,57 & 0,90 & 0,21 & 0,08 & 1,21 & 0,89 & 6,16 & 136,93 & 0,89 \\
\hline $\mathrm{s}(\hat{m})$ & 0,26 & 0,03 & 0,23 & 0,37 & 0,07 & 0,03 & 0,49 & 0,37 & 2,51 & 55,90 & 0,37 \\
\hline LI & 15,54 & 1,00 & 10,62 & 10,79 & 1,68 & 1,26 & 12,06 & 9,06 & 29,04 & 831,30 & 12,06 \\
\hline LS & 16,86 & 1,16 & 11,82 & 12,68 & 2,02 & 1,44 & 14,60 & 10,94 & 41,96 & 1118,70 & 13,94 \\
\hline $\mathrm{E}$ & 4,10 & 7,29 & 5,34 & 8,03 & 9,11 & 6,50 & 9,53 & 9,39 & 18,20 & 14,74 & 7,22 \\
\hline $\mathrm{CV}$ & 3,90 & 6,95 & 5,09 & 7,65 & 11,21 & 6,20 & 9,08 & 8,94 & 17,34 & 14,04 & 6,88 \\
\hline \multicolumn{12}{|l|}{20 Plantas } \\
\hline$m$ & 15,53 & 1,10 & 11,53 & 11,95 & 1,70 & 1,40 & 13,33 & 11,00 & 38,33 & 1058,33 & 13,67 \\
\hline $\mathrm{s}^{2}$ & 0,17 & 0,00 & 0,28 & 0,56 & 0,01 & 0,00 & 1,47 & 0,80 & 34,27 & 6416,67 & 0,67 \\
\hline $\mathrm{s}$ & 0,41 & 0,06 & 0,53 & 0,75 & 0,11 & 0,06 & 1,21 & 0,89 & 5,85 & 80,10 & 0,82 \\
\hline $\mathrm{s}(\hat{m})$ & 0,17 & 0,03 & 0,22 & 0,31 & 0,03 & 0,03 & 0,49 & 0,37 & 2,39 & 32,70 & 0,33 \\
\hline LI & 15,10 & 1,03 & 10,98 & 11,17 & 1,61 & 1,33 & 12,06 & 10,06 & 32,19 & 974,27 & 12,81 \\
\hline LS & 15,96 & 1,17 & 12,09 & 12,73 & 1,79 & 1,47 & 14,60 & 11,94 & 44,48 & 1142,40 & 14,52 \\
\hline E & 2,76 & 6,03 & 4,61 & 6,57 & 5,24 & 4,74 & 9,53 & 8,53 & 16,03 & 7,94 & 6,27 \\
\hline $\mathrm{CV}$ & 2,63 & 5,75 & 4,84 & 6,26 & 6,44 & 4,52 & 9,08 & 8,13 & 15,27 & 7,57 & 5,97 \\
\hline \multicolumn{12}{|l|}{40 Plantas } \\
\hline$m$ & 15,03 & 1,07 & 11,25 & 11,87 & 1,77 & 1,32 & 13,67 & 9,00 & 40,50 & 1016,67 & 12,67 \\
\hline$s^{2}$ & 0,10 & 0,00 & 0,19 & 0,35 & 0,01 & 0,00 & 1,07 & 0,40 & 1,90 & 5666,67 & 0,27 \\
\hline $\mathrm{s}$ & 0,32 & 0,05 & 0,44 & 0,59 & 0,08 & 0,04 & 1,03 & 0,63 & 1,38 & 75,28 & 0,52 \\
\hline $\mathrm{s}(\hat{m})$ & 0,13 & 0,02 & 0,18 & 0,24 & 0,03 & 0,02 & 0,42 & 0,26 & 0,56 & 30,73 & 0,21 \\
\hline LI & 14,70 & 1,01 & 10,79 & 11,25 & 1,70 & 1,27 & 12,58 & 8,34 & 39,05 & 937,67 & 12,12 \\
\hline LS & 15,37 & 1,12 & 11,71 & 12,49 & 1,83 & 1,36 & 14,75 & 9,66 & 41,95 & 1095,67 & 13,21 \\
\hline E & 2,24 & 5,08 & 4,08 & 5,24 & 3,76 & 3,25 & 7,93 & 7,37 & 3,57 & 7,77 & 4,28 \\
\hline $\mathrm{CV}$ & 2,13 & 4,84 & 3,88 & 4,99 & 4,62 & 3,10 & 7,56 & 7,03 & 3,40 & 7,40 & 4,08 \\
\hline
\end{tabular}

$\hat{m}$ é a estimativa da média amostral; $\mathrm{s}^{2}$ é a variância; s é o desvio-padrão; s $(\hat{m})$ é o erro-padrão da média; LI é o limite inferior da distribuição $t$ de Student, a $5 \%$ de probabilidade; LS é o limite superior da distribuição $t$ de Student, a $5 \%$ de probabilidade; E é a porcentagem de erro em relação à média, e CV é o coeficiente de variação. 
Madgwick (1964) observou, em florestas, que os maiores teores foliares e as maiores variações de nutrientes ocorrem em árvores sombreadas. Entretanto, há menor amplitude de variação de nutrientes entre árvores dominantes e co-dominantes, provavelmente por estas não estarem sombreadas (Lowry \& Avard, 1969). De maneira geral, para o diagnóstico foliar, Mead (1984) recomenda coletar amostras em 20 árvores, sejam elas dominantes ou co-dominantes em florestas de eucalipto.

Admitindo-se um erro aceitável de 5 a $10 \%$, conforme proposto por Lamb (1976) e Mead (1984), 10 plantas de mangueira foram suficientes para a análise dos macronutrientes (Figura 1a). Porém, para os micronutrientes, foram necessárias, no mínimo, 20 plantas e, se considerar o Fe, a amostra deve ter, pelo menos, 30 plantas para manter o erro menor que $10 \%$ (Figura $1 \mathrm{~b}$ ).

Amplas variações nos teores foliares de Fe são comuns nesse tipo de análise, também relatadas por Lamb (1976) em Eucalipto e, em Goiabeira, por Dahiya \& Joon (1995). Tais oscilações nas análises, especialmente em solos tropicais, podem ocorrer devido à alta quantidade de óxidos de ferro presentes no solo do pomar.

\section{CONCLUSÃO}

Para as determinações químicas dos macronutrientes, a coleta de uma folha em cada um dos quatro pontos cardeais em 10 plantas de mangueira foram suficientes. Já para os micronutrientes, foram necessárias, no mínimo, 20 plantas e, se considerar o $\mathrm{Fe}$, a amostra deverá conter, pelo menos, 30 plantas.

\section{REFERÊNCIAS}

AVILAN, L.A.R.; CARMELO, R.A. EI mango. Caracas: Editorial America, 1990.401p.

BATAGLIA, O.C.; FURLANI, A.M.C.; TEIXEIRA, J.P.F.; FURLANI, P.R.; GALLO, J.R. Métodos de análise química de plantas. Campinas: Instituto Agronômico de Campinas, 1983. 48p. (Boletim Técnico, 78)

DAHIYA, S.S.; JOON, M.S. Variation in mineral composition of leaves of guava cultivar L-49 as affected by sample size. Crop Research, Edinburg, v.9, n.1, p.121-122 1995.

EMBRAPA. EMPRESA BRASILEIRA DE AGROPECUÁRIA. Centro Nacional de Pesquisa de Solo. Sistema brasileiro de classificação de solos. Brasília: Produção de Informações, 1999. 412p.

GUIMARÃES, P.T.G. Nutrição e adubação da mangueira. Informe Agropecuário, Belo Horizonte, v.8, n.86,p.28-35,1982.

HOLLAND, D.A.; LITTLE, R.C.; ALLEN, M.; DERMOTT, W. Soil and leaf sampling in apple orchards. Journal of Horticultural Science, Ashford, v. 42, p. 403-417, 1967.

KLEIN, I. Growth and mineral accumulation of apple tress. Acta Horticulturae, Wageningen, v. 92, p.298, 1980.

KNIGHT, PJ. Fertilizer practice in New Zealand forest nurseries. Journal Forestry Science, Bengladish, , v.8, n.1, p, 351-368, 1978.

LAMB, D. Variations in the foliar concentrations of macro and micro elements in a fast-growing tropical eucalypt. Plant and Soil, Netherlands, v.45, p.477-492. 1976.

LIRIO, V.S. Panorama econômico da cultura e comercialização da manga. In: ROZANE, D.E.; DAREZZO, R.J.; AGUIAR, R.L.; AGUILERA, G.H.A.;ZAMBOLIM, L. (Eds.) Manga: produção integrada, industrialização e comercialização. Viçosa: Universidade Federal de Viçosa UFV, 2004. p.1-16.

LOWRY, G. L.; AVARD, P. M. Nutrient content of black spruce and jack pine needles: III. Seasonal variations and recommended sampling procedures. Point Clare: Research Institute of Canada, 1969. 54p. (Woodl,.10)

MADGWICK, H.A.I. Variations in the chemical composition of red pine (Pinus resinosa Ait) leaves:.a comparison of well grown and poorly grown trees. Forestry, Oxford, v.37, n.1, p. $87-94,1964$.

MALAVOLTA, E. ABC da análise de solos e folhas. Piracicaba: Agronômica Ceres, 1992. 124p.

MALAVOLTA, E.; VITTI, G.C.; OLIVEIRA, S.A. Avaliação do estado nutricional das plantas: princípios e aplicações. Piracicaba: POTAFÓS, 1997.319p.

MARSCHNER, H. Mineral nutrition of higher plants. $2^{\text {nd }}$ ed. San Diego: Academic, 1995. 902p.

MEAD, D.J. Diagnosis of nutrient deficiencies in plantations. In: BOWEN, G.D.; NAMBIAR, E.K. (Ed.) Nutrition of plantation forests. London: Academic Press, 1984. cap. 10, p.259-291.

MEDEIROS, A.A.; AMORIM, J.R.A.; SILVA, D.J.; DANTAS, J.A.; GUERRA, A.G. Mineral composition of leaves and fruits of irrigated mango trees in Rio Grande do Norte State, Brazil. Acta Horticulturae, Wageningen, n. 645, p.403-410, 2004.

NATALE, W.; COUTINHO, E.L.M.; BOARETTO, A E.; BANZATTO, D. A. Influência da época de amostragem na composição química das folhas de goiabeira (Psidium guajava L.). Revista de Agricultura, Piracicaba, v. 69, p. 247255, 1994.

ORLANDO FILHO, J.; CAMPOS, H. Número ideal de folhas para a diagnose foliar em cana-de-açúcar (cana-planta). Brasil Açucareiro, Rio de Janeiro, v.85, n.1, p.10-17, 1975a.

ORLANDO FILHO, J.; CAMPOS, H. Número ideal de folhas para a diagnose foliar em cana-de-açúcar (soqueira). Brasil Açucareiro, Rio de Janeiro, v.85, n.4, p.23-28, 1975 b.

PINTO, A.C.Q. Melhoramento genético da manga (Mangifera Indica L.) no Brasil. In: ROZANE, D.E.; DAREZZO, R.J.; AGUIAR, R.L.; AGUILERA, G.H.A.; ZAMBOLIM, L. (Eds.) Manga: produção integrada, industrialização e comercialização. Viçosa: Universidade Federal de Viçosa, 2004. p.17-78.

PONCHNER, S.; ROJAS,R.; BORNEMISZA, E. Variación Estacional de Nutrimentos en Arboles de Mango ( Mangifera indica) en tres suelos del Pacifico Seco de Costa Rica. Imacronutrientes. Agronomia Costarricense, San José, v.17, n.2, p.21-30, 1993.

PRADO, R.M. Nutrição e desordens fisiológicas na cultura da manga. In: ROZANE, D.E.; DAREZZO, R.J.; AGUIAR, R.L.; AGUILERA, G.H.A.;ZAMBOLIM, L. (Eds.) Manga: produção integrada, industrialização e comercialização. Viçosa: Universidade Federal de Viçosa, 2004. p.199-232.

QUAGGIO, J.A.; SOARES, N.B.; FURLANI, P.R. RAIJ, B.van, PIZA JR. C. De T.; PINTO, A.C. de Q. Recomendações de 
adubação e calagem para o Estado de São Paulo. 2.ed. Campinas: Instituto Agronômico \& Fundação IAC, 1977. p.121-125. (Boletim Técnico, 100)

RAIJ, B. van; ANDRADE, J.C.;. CANTARELLA H.; QUAGGIO, J.A. Análise química para avaliação da fertilidade de solos tropicais. Campinas: Instituto Agronômico, 2001, 285p.

RAIJ, B.van, CANTARELLA, H., QUAGGIO, J.A, FURLANI, A.M.C. (Eds.) Recomendações de adubação e calagem para o Estado de São Paulo. 2.ed. Campinas: Instituto Agronômico \& Fundação IAC, 1997.p.121-125. (Boletim Técnico, 100)

SIQUEIRA, D.L.; MOTOIKE, S.Y.; SALOMÃO, L.C.C. Cultura da Mangueira. Viçosa: Editora UFV, 1999. 47p.

SODRÉ, G.A.; MARROCOS, P.C.L.; CHEPOTE, R.E.; PACHECO, R.G. Uso do desvio- padrão para estimativa do tamanho da amostra de plantas de cacau (Theobroma cacao L.) em estudos de nutrição. Agrotrópica, Ilhéus, v. 13, n. 3, p. 145$150,2001$.

THAKUR, R.S.; RAO, P.G.S.; CHADHA, K.L.; SAMRA, J.S. Variation in mineral composition of mango leaves as contributed by leaf sampling factors. Communication in Soil Science and Plant Analysis, Monticello, v.12,p.331-343,1981. 